\title{
Mammalian P5CR and P5CDH: Protein Structure and Disease Association
}

\author{
Chien-An A. $\mathrm{Hu}^{1 *}$ and Yongqing $\mathrm{Hou}^{2}$ \\ ${ }^{1}$ Department of Biochemistry and Molecular Biology, University of New Mexico Health Sciences Center, Albuquerque, \\ New Mexico 87131-0001, USA \\ ${ }^{2}$ School of Animal Science and Nutritional Engineering, Wuhan Polytechnic University, Wuhan, Hubei, 430023, P. R. China
}

Received: September 01, 2014; Accepted: October 20, 2014; Published: October 30, 2014

*Corresponding author: Chien-An A. Hu, MSC08 4670, Department of Biochemistry and Molecular Biology, UNM HSC, Albuquerque, NM87131, USA, Tel: +505-272-8816; E-mail: AHu@salud.unm.edu

\begin{abstract}
The interconversions of L-proline (Pro) and L-glutamate (Glu) in mammalian cells involve an obligatory intermediate, $\Delta^{1}$-pyrroline5-carboxylate (P5C/PYC), and four uni-directional enzymes, proline oxidase/dehydrogenase (POX/PRODH), P5C dehydrogenase (P5CDH), P5C synthase (P5CS/PYCS), and P5C reductase (P5CR/PYCR). The catabolism of Pro by two dehydrogenation reactions catalyzed by POX and $\mathrm{P} 5 \mathrm{CDH}$ is an important source of oxidizing signaling, whereas the anabolism of Pro through two reduction reactions catalyzed by P5CS and P5CR maintains redox homeostasis to promote cell growth. In addition, Pro is one of the "conditionally essential" amino acids in the neonatal intestine in both humans and animals. The homeostatic balance of Pro, Glu, and Arginine is critical for the growth, redox balance, immunomodulation, and development of mammals. Recent discoveries strongly suggest that Pro metabolic enzymes are tightly regulated by spatial-temporal gene expression, tissue and cellspecificity, substrate and/or inhibitor abundance, and subcellular compartmentalization. In terms of disease association, mutations in human P5CR1 gene have been identified in patients with autosomal recessive Cutis laxa type IIB and type IIIB/De Barsy syndrome, whereas mutations in $\mathrm{P} 5 \mathrm{CDH}$ gene cause type II hyperprolinemia. Importantly, $\mathrm{x}$-ray crystallographic studies have revealed the protein structures of human P5CR 1 and mammalian P5CDH. These new discoveries in structure function relationship may provide crucial guidelines in the treatment of the corresponding disorders.
\end{abstract}

Keywords: Cutis laxa Type IIB and Type IIIB; De Barsy syndrome; DJ-1; ORAOV1; P5CR/PYCR; P5CDH; Subcellular localization

\section{Abbreviations}

P5C/PYC: $\quad \Delta{ }^{1}$-pyrroline-5-carboxylate; P5CDH: $\quad$ P5C Dehydrogenase; P5CR: P5C Reductase; P5CS: P5C Synthase; OAT: Ornithine Aminotransferase; GSA: Glutamic- $\gamma$-Semialdehyde

\section{Introduction}

In mammals, L-proline (Pro) and L-glutamate (Glu) are interconverted by four highly regulated, uni-directional enzymes, namely, Pro oxidase/dehydrogenase (POX/PRODH), $\Delta^{1}$-pyrroline-5-carboxylate (P5C/PYC), P5C Dehydrogenase (P5CDH), P5C Synthase (P5CS/PYCS), and P5C Reductase (P5CR/ PYCR), with P5C as the obligatory intermediate[1-4] (Table 1).
P5C is in tautomeric equilibrium with Glutamic- $\gamma$-Semialdehyde (GSA), which is reduced to Pro by the cytosolic and mitochondrial NAD(P)H-dependent P5CR isozymes. In addition, P5C/GSA is a substrate for two other enzymes, mitochondrial $\mathrm{P} 5 \mathrm{CDH}$, which converts P5C/GSA to Glu [5], and mitochondrial Ornithine (Orn) Aminotransferase (OAT), which catalyzes the interconversion of P5C and Orn [6]. Orn enters the urea cycle mainly for ammonia detoxification and Arginine (Arg) biosynthesis (Figure 1). It has been shown that Pro and Arg are two of the "conditionally essential" amino acids in the neonatal intestine of mammals. The interconversions of Pro, Orn, Glu, and Arg are critical for the growth and immunomodulation of mammals $[1,4,7]$. At the cellular level, the catabolism/degradation of Pro by POX and then $\mathrm{P} 5 \mathrm{CDH}$ is an important source of redox signaling, whereas the anabolism/biosynthesis of Pro through P5CS and then P5CR maintains redox homeostasis to promote cell growth. In this review, we summarize the recent discoveries on human P5CR isozymes and P5CDH and their associated diseases/disorders.

\section{Human P5CR: Three isozymes encoded by three different genes}

Three isozymes of human P5CR/PYCR (EC1.5.1.2), have been identified, cloned, and characterized $[1,2,8]$. Human P5CR1, P5CR2, and P5CRL are encoded by three different genes, localized at three different chromosomal locations (Table 1). These three isozymes catalyze ATP and NAD(P)H-dependent reduction of $\mathrm{P} 5 \mathrm{C}$ to Pro, which is important for the transfer of oxidizing potential across the cell $[9,10]$. The human P5CR1/ PYCR1 structural gene [also known as Proliferation-Inducing protein 45 (PIG45)] is localized on chromosome 17q25.3, which encodes two protein isoforms, a 319-amino acid residue (aa) and a 316-aa polypeptide, respectively. The human P5CR2/ PYCR2 structural gene is localized on chromosome 1q42.12, which encodes two protein isoforms, a 320-aa and a 246-aa polypeptide, respectively. Recently, De Ingeniis and colleagues [8] confirmed that there is a new isozyme of P5CR, P5CRL, in melanoma cells. The human P5CRL/PYCR3 structural gene is localized on chromosome 8q24.3, which encodes two isoforms, a 286-aa and a 266-aa polypeptide, respectively. In addition, it has 
Table 1: Human P5CR Isozymes and P5CDH: from genes to protein isozymes to associated disorders.

\begin{tabular}{|c|c|c|c|c|c|c|}
\hline Enzyme & Gene Name & Gene ID & Map Location & oMIM\# & Isozyme & \# Amino Acids \\
\hline P5CR1 & PYCR1/P5CR1 & 5831 & $17 q 25.3$ & 179035 & P5CR1.1 & 319 \\
\hline & & & & 612940 & P5CR1.2 & 316 \\
\hline & & & & 614438 & & \\
\hline P5CR2 & PYCR2/P5CR2 & 29920 & $1 \mathrm{q} 42.12$ & N/A & P5CR2.1 & 320 \\
\hline P5CRL/P5CR3 & PYCRL/P5CR3 & 65263 & $8 \mathrm{q} 24.3$ & N/A & P5CRL.1 & 246 \\
\hline & & & & & P5CRL.2 & 266 \\
\hline P5CDH & ALDH4A1/P5CDH & 8659 & $1 \mathrm{p} 36$ & 606811 & P5CDH & 563 \\
\hline
\end{tabular}

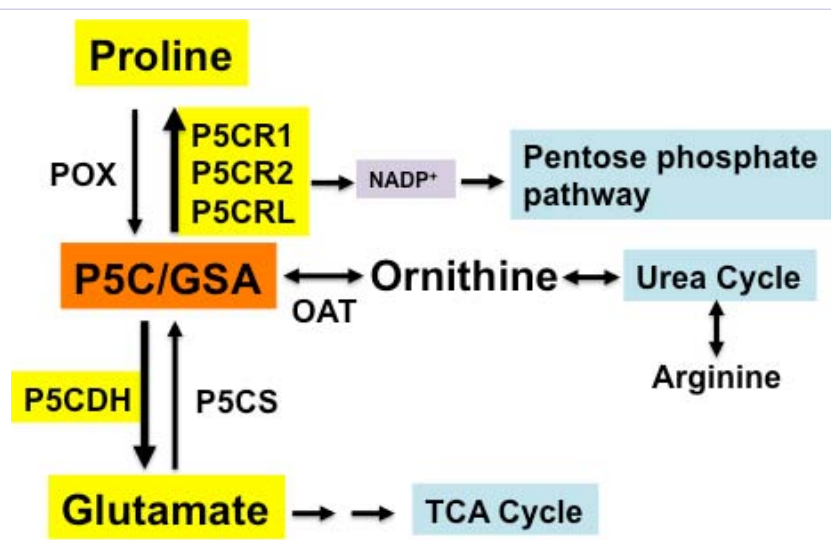

Figure 1: The interconversions of proline, glutamate, and ornithine. This tri-amino-acid-cycle also links with the urea cycle and the pentose phosphate pathway.

been demonstrated that P5CR1 and P5CR2 are localized in the mitochondria and are primarily involved in conversion of Glu to Pro, whereas P5CRL is localized in the cytosol and is exclusively linked to the conversion of Orn to Pro, and is not feedback inhibited by proline. Previously, Merrill and colleagues [11] showed that human P5CR in erythrocytes not only catalyzes the obligatory step in Pro biosynthesis, but also plays a physiological role in the generation of $\mathrm{NADP}^{+}$. The normal abundance of P5CR in the cell is maintained relatively low due to its high turnover [9]. A recent study by Krishnan and colleagues [12] showed over expression of P5CR1 resulted in 2-fold higher proline content, significantly lowered free radical levels, and increased cell survival. Another studies showed that increased P5CR1 activity was measurable in pulmonary and colorectal tumors $[13,14]$. In contrast, mammalian P5CR2 and P5CRL are relatively new and not well studied.

With regard to their interactomes, interestingly, PYCR1 has been identified as an interacting protein of two important regulatory proteins, DJ-1 [15] and Oral Cancer Over expressed 1 (ORAOV-1) [16]. DJ-1, encoded by the DJ-1/PARK7 gene, plays various functions involved in transcriptional regulation, antioxidative activity, and regulation of mitochondrial complex I. It has been shown that DJ-1 and PYCR1 interacts and colocalizes in mitochondria. DJ-1 increases the enzymatic activity of PYCR1 in vitro [15]. ORAOV1, encoded by ORAOV1 gene, is frequently amplified in esophageal squamous cell cancer, and has been shown to regulate the cell cycle, apoptosis and angiogenesis. Cancer cells overexpressing ORAOV1 exhibited significantly increased tumorigenicity and larger tumors with poor differentiation. It has been shown that ORAOV1 also increases the enzymatic activity of PYCR1 and the production of Pro when it binds with P5CR1 [16]. However, whether P5CR1, DJ-1, and ORAOV1 interact with each other and function in the same complex in certain cell types is not known.

\section{Human P5CR1 Protein structure and associated diseases}

The crystal structure of human P5CR1 have been reported recently $[13,14]$. The 2.8 Angstroms $(\AA)$ resolution structure of the P5CR1 apo enzyme and its 3.1 A resolution ternary complex with NAD(P)H and substrate-analog demonstrated that human P5CR1 possesses a decameric architecture with five homodimer subunits. It has been hypothesized that human P5CR1 possesses ten catalytic sites arranged around a peripheral circular groove. In terms of disease association, homozygous or compound heterozygous mutations in human P5CR1 have been identified in autosomal recessive Cutis laxa, Type IIB (ARCL2B or Cutis laxa with progeroid features; OMIM \#612940; Table 1) [17-19]. The clinical phenotype of ARCL2B includes cutis laxa, abnormal growth and development, and associated skeletal abnormalities [18]. In addition, P5CR1 mutations have been linked to autosomal recessive cutis laxa type III (ARCL3, OMIM \#614438), also known 
as De Barsy syndrome. ARCL3 is a rare autosomal recessive disorder, characterized by an aged appearance with distinctive facial features, sparse hair, ophthalmologic abnormalities, intrauterine growth retardation, and cutis laxa.

\section{Human P5CDH: Protein structure and associated disease}

Mammalian P5CDH (EC 1.5.1.12) is a mitochondrial matrix $\mathrm{NAD}^{+}$-dependent dehydrogenase which converts P5C/GSA to Glu, and thus is a high $\mathrm{Km} /$ low affinity Aldehyde Dehydrogenase (ALDH) with GSA as a primary substrate. Mammalian P5CDH also exhibits activity with other aldehydes, and is dubbed as ALDH4A1 (ALDH, family 4, subfamily A, member 1) [1,2] (Table 1). Human $\mathrm{P} 5 \mathrm{CDH} / \mathrm{ALDH} 4 \mathrm{~A} 1$ structural gene is localized on chromosome 1 p36 and encodes a 563-aa polypeptide [1,5]. Importantly, it has been demonstrated in plants that Lack of P5CDH activity led to higher ROS production in the presence of Pro excess. Therefore, oxidation of $\mathrm{P} 5 \mathrm{C}$ to Glu by $\mathrm{P} 5 \mathrm{CDH}$ is critical to prevent P5C-Pro intensive cycling and avoid ROS production from electron run-off [20-22]. In additional, it has been shown that Drosophila deficient in $\mathrm{P} 5 \mathrm{CDH}$ showed hyperprolinemia, swollen mitochondria, and early embryonic lethality [23]. Taken together, these observations suggest that $\mathrm{P} 5 \mathrm{CDH}$ plays a protective role against ROS generation, mitochondria and cell damage, and apoptosis.

To understand the functions of $\mathrm{P} 5 \mathrm{CDH}$ at the molecular level, how substrates and inhibitors interact with the enzyme, and how the substituted residues encoded by the mutant alleles of $\mathrm{P} 5 \mathrm{CDH}$ can affect the enzymatic activity, the crystal structures of human and mouse P5CDH were determined recently [24,25] . Both wildtype $\mathrm{P} 5 \mathrm{CDH}$ and mutant $\mathrm{P} 5 \mathrm{CDH}$ proteins carrying S352A (2.4 $\AA$ ) and S352L (2.85 ̊) substitutions were resolved. In addition, $2.5-\AA$ resolution Structures of the mouse P5CDH complexed with sulfate ion (1.3 ̊̊ resolution), glutamate (1.5 $)$ ), and $\mathrm{NAD}^{+}(1.5 \AA)$ were determined in order to obtain highresolution views of the active site. Together, the structures showed that single amino acid substitutions cause structural alterations and enzyme inactivation. Interestingly, the structureactivity relationship demonstrated that the semialdehyde carbon chain length and the position of the aldehyde group in relation to the cysteine nucleophile and oxyanion hole of mouse P5CDH are critical. Efficient 4- and 5-carbon substrates share the common feature of being long enough to span the distance between the anchor loop at the bottom of the active site and the oxyanion hole at the top of the active site. The inactive 2- and 3-carbon semialdehydes bind the anchor loop but are too short to reach the oxyanion hole [25]. The $\mathrm{K}_{\mathrm{i}}$ values are $0.27 \mathrm{mM}$ for glyoxylate, $58 \mathrm{mM}$ for succinate, $30 \mathrm{mM}$ for glutarate, and 12 $\mathrm{mM}$ for L-glutamate. Interestingly, malonate is not an inhibitor [25]. With regard to its disease association, deficiency of P5CDH causes type II Hyperprolinemia (HPII), an autosomal recessive disorder characterized by accumulation of P5C and Pro [6,26,27]. Although HPII has been considered as a benign disorder, further research indicated that HPII may cause clinical manifestations, such as childhood febrile seizures.

\section{Acknowledgements}

This work was supported, in part, by the pilot projects (\#0302 and \#0224 to CAAH) of UNM CTSC grant (8UL1TR000041).

\section{References}

1. Phang JM, Hu CA, Valle D. Disorders of proline and hydroxyproline metabolism. In: CR Scriver, AL Beaudet, WS Sly, D Valle, Metabolic and Molecular Basis of Inherited Disease. New York: McGraw Hill Press; 2001. 1821-1838.

2. Hu CA, Bart Williams D, Zhaorigetu S, Khalil S, Wan G, Valle D. Functional genomics and SNP analysis of human genes encoding proline metabolic enzymes. Amino Acids. 2008; 35(4): 655-64. doi: 10.1007/s00726-008-0107-9.

3. Hu CA, Khalil S, Zhaorigetu S, Liu Z, Tyler M, Wan G, Valle D. Human Delta1-pyrroline-5-carboxylate synthase: function and regulation. Amino Acids. 2008; 35(4): 665-72. doi: 10.1007/s00726-008-0075-0.

4. Wu G, Bazer FW, Burghardt RC, Johnson GA, Kim SW, Knabe DA, et al. Proline and hydroxyproline metabolism: implications for animal and human nutrition. Amino Acids. 2011; 40(4): 1053-1063. doi: 10.1007/ s00726-010-0715-z.

5. $\mathrm{Hu} \mathrm{CA}$, Lin WW, Valle D. Cloning, characterization and expression of cDNAs encoding human delta 1-pyrroline-5-carboxylate dehydrogenase. J Biol Chem. 1996; 271(16): 9795-9800.

6. Valle D, Goodman SI, Harris SC, Phang JM. Genetic evidence for a common enzyme catalyzing the second step in the degradation of proline and hydroxyproline. J Clin Invest. 1979; 64(5):1365-1370.

7. Ren W, Zou L, Ruan Z, Li N, Wang Y, Peng Y, et al. Dietary L-proline supplementation confers immunostimulatory effects on inactivated Pasteurella multocida vaccine immunized mice. Amino Acids. 2013; 45(3): 555-61. doi: 10.1007/s00726-013-1490-4.

8. De Ingeniis J, Ratnikov B, Richardson AD, Scott DA, Aza-Blanc P, De SK, et al. Functional specialization in proline biosynthesis of melanoma. PLoS One. 2012; 7(9). e45190.

9. Phang JM. The regulatory functions of proline and pyrroline-5carboxylic acid. Curr Top Cell Regul. 1985; 25: 91-132.

10. Dougherty KM, Brandriss MC, Valle D. Cloning human pyrroline-5carboxylate reductase cDNA by complementation in Saccharomyces cerevisiae. J Biol Chem. 1992; 267(2): 871-875.

11. Merrill MJ, Yeh GC, Phang JM. Purified human erythrocyte pyrroline-5carboxylate reductase. Preferential oxidation of NADPH. J Biol Chem. 1989; 264(16): 9352-9358.

12. Krishnan N, Dickman MB, Becker DF. Proline modulates the intracellular redox environment and protects mammalian cells against oxidative stress. Free Radic Biol Med. 2008; 44(4): 671-681.

13. Meng Z, Lou Z, Liu Z, Hui D, Bartlam M, Rao, Z. Purification, characterization, and crystallization of human pyrroline-5-carboxylate reductase. Protein Expr Purif. 2006; 49(1): 83-87.

14. Meng Z, Lou Z, Liu Z, Li M, Zhao X, Bartlam M, et al. Crystal Structure of Human Pyrroline-5-carboxylate Reductase. J Mol Biol. 2006; 359(5): 1364-1377.

15. Yasuda T, Kaji Y, Agatsuma T, Niki T, Arisawa M, Shuto S, et al. DJ-1 cooperates with PYCR1 in cell protection against oxidative stress. Biochem Biophys Res Commun. 2013; 436(2): 289-294. doi: 10.1016/j.bbrc.2013.05.095. 
16. Togashi Y, Arao T, Kato H, Matsumoto K, Terashima M, Hayashi H, et al. Frequent amplification of ORAOV1 gene in esophageal squamous cell cancer promotes an aggressive phenotype via proline metabolism and ROS production. Oncotarget. 2014; 5(10): 2962-73.

17. Morava E, Guillard M, Lefeber DJ, Wevers RA. Autosomal recessive cutis laxa syndrome revisited. Eur J Hum Genet. 2009; 17(9), 1099 1110. doi: 10.1038/ejhg.2009.22.

18. Guernsey DL, Jiang H, Evans S C, Ferguson M, Matsuoka M, Nightingale $\mathrm{M}$, et al. Mutation in pyrroline-5-carboxylate reductase 1 gene in families with cutis laxa type 2. Am J Hum Genet. 2009; 85(1): 120-129. doi: 10.1016/j.ajhg.2009.06.008.

19. Reversade B, Escande-Beillard N, Dimopoulou A, Fischer B, Chng SC, L Y, et al. Mutations in PYCR1 cause cutis laxa with progeroid features. Nat Genet. 2009; 41(9): 1016-1021. doi: 10.1038/ng.413.

20. Deuschle K, Funck D, Hellmann H, Däschner K, Binder S, Frommer WB. A nuclear gene encoding mitochondrial Delta-pyrroline-5-carboxylate dehydrogenase and its potential role in protection from proline toxicity. Plant J. 2001; 27(4): 345-56.

21. Deuschle K, Funck D, Forlani G, Stransky H, Biehl A, Leister D, et al. The role of [Delta]1-pyrroline-5-carboxylate dehydrogenase in proline degradation. Plant Cell. 2004; 16(12): 3413-25.

22. Miller G, Honig A, Stein H, Suzuki N, Mittler R, Zilberstein A. Unraveling delta1-pyrroline-5-carboxylate-proline cycle in plants by uncoupled expression of proline oxidation enzymes. J Biol Chem. 2009; 284(39): 26482-92. doi: 10.1074/jbc.M109.009340.

23. He F, DiMario PJ. Drosophila delta-1-pyrroline-5-carboxylate dehydrogenase (P5CDh) is required for proline breakdown and mitochondrial integrity-Establishing a fly model for human type II hyperprolinemia. Mitochondrion. 2011; 11(3): 397-404. doi: 10.1016/j.mito.2010.12.001.

24. Srivastava D, Singh RK, Moxley MA, Henzl MT, Becker DF, Tanner JJ. The three-dimensional structural basis of type II hyperprolinemia. J Mol Biol. 2012; 420(3):176-89. doi: 10.1016/j.jmb.2012.04.010.

25. Pemberton TA, Tanner JJ. Structural basis of substrate selectivity of $\Delta(1)$-pyrroline-5-carboxylate dehydrogenase (ALDH4A1): semialdehyde chain length. Arch Biochem Biophys. 2013; 538(1):3440. doi: 10.1016/j.abb.2013.07.024.

26. Flynn MP, Martin MC, Moore PT, Stafford JA, Fleming GA, Phang JM. Type II hyperprolinaemia in a pedigree of Irish Travellers (nomads). Arch Dis Child. 1989; 64(12): 1699-1707.

27.Geraghty MT, Vaughn D, Nicholson AJ, Lin WW, Jimenez-Sanchez $\mathrm{G}$, Obie $\mathrm{C}$, et al. Mutations in the delta-1-pyrroline 5-carboxylase dehydrogenase gene cause type II hyperprolinemia. Hum Mol Genet. 1998; 7(9): 1411-1415. 